\title{
The Extract of the Coal and the Design of the Reprocessing Mechanic Equipment
}

\author{
Hongyan Shi \\ Inner Mongolia University for The Nationlities, Tongliao, 028000, China
}

Keywords: Coal. Extract. Reprocess. Mechanic equipment

\begin{abstract}
Coal is one of the major energy of people`s daily life and producing work, but because burning the coal will create some harmful gases, so it is necessary to extract and reprocess the coal before using it. This paper first analyzes the property of the coal, and then states the reason and theory of the coal reprocessing, clarify the harm of the direct use of the coal, and make sure that the selection, desulphuration and carbonization is needed, besides, decide the design philosophy of the coal extracting and reprocessing mechanic equipment.
\end{abstract}

\section{Introducing}

Coal is the most common energy among all the industrial energy, it enjoys the same position as the natural gases and petrol, and it occupies a broader market space to a greater extent. As one of the major energy, it is most commonly used in combustion,heating in the civil and industry etc. Because most of the main mining source of the coal is the coal mine, so there must be a lot of foreign substance. Among them, the sulfur is the major hazardous substances, the burning process will create sulfur dioxide, that will harm the atmosphere and lead to the acid rain. Besides,there is saltpeter exist in the coal, that would not only burn but also trigger the explosion. So,the coal from the coal mine can be used as a energy only after some processing work. The extracting and processing of the coal need necessary mechanic equipment. This paper states the principle of coal extracting and processing, and analyzes the design of the processing equipment.

\section{The property analysis of the coal}

The coal is a nonrenewable resources, although its storage on the earth is very abundant, but as people`s exploitation and the usage requirement s expanding, the coal resource is sure to be less and less. The main chemical element of the coal is the carbon, carbon is flammable, it can release the heat when burning in a high temperature environment. Besides, there will be no due processing during the exploitation, so there will be many unqualified or low-quality coal. And this coal may not be sufficient burned, it will not only waste the energy but also fail to meet the expected energy supply.

\section{The extraction and reprocessing of the coal}

The major element of the coal is carbon, there is sulfur element existed in part of the coal.and this is the basic chemical property of coal, its physical texture is very hard, which makes it suitable for continued burning, the heat it release is not very much,the firing duration is very long, and the combustion waste can be used as coke. The production of the coal is very abundant,and it is suitable for home-use,civil and industry. The coal has a very broad application range, and it effects the natural environment to some degree. So, the coal can only be used after extraction and processing.

\section{Problem analysis during the usage of the coal}

There will be some problems when using the coal, and that can be analyzed from two aspects:

Firstly, during the daily usage of the coal, it is very likely to produce much smoke. In the air pollution, there is certain proportion about the smoke caused by the coal in the air. Because there will be decomposition in the coal`s long -term`s storage, it will cause some ashes which will effect the surrounding environment. 
Secondly, there are a lot of problems during the coal's burning, first, the main element of the coal is carbon, and it will release carbon dioxide during the burning. The carbon dioxide is not a toxic gas, but too much of it will lead to greenhouse effect. Secondly, there is sulfur in the coal.which will release sulfur dioxide in the burning process, which will cause some air pollution if it was released into the air. Sulfur dioxide is harmful gases, it will lead to the acid rain, which will cause certain damage to people and the building. At last, the coal in the market are of different quality. The coal of different quality has different burning efficiency, additionally, due to the low-quality of some coal, it will result to the incomplete combustion, the carbon monoxide is poisonous, and it will cause great harm to the environment.

\section{The analysis of the reason of coal's extraction and reprocessing}

From the problem mentioned above, we can conclude clearly that the burning of the carbon will produce a lot of carbon dioxide, besides because of the sulfur element, the gas released will contain the sulfur dioxide, which will have enormous effect to the environment. In addition, the incomplete burning of the coal will have enormous bad effect, carbon monoxide is poisonous, and it is harmful to the human life and producing. Besides, the incomplete burning will lead to some waste. Bused on the several points of reason mentioned above, the coal is in need of extraction to improve its purity and further reprocessing to reduce the sulfur inside.

\section{The measure of the coal's extraction and reprocessing}

After the analysis above, the major measures of the coal`s extraction and reprocessing are as following:

First, selecting the carbon`s quality is to classifying the carbon. The higher the quality of the coal combustion is, the more fully the coal will burn, thus to reduce the probability of the carbon monoxide, and it would provide more energy from the same quality of coal.

Secondly, conduct desulfurization treatment, the sulfur will exist in the coal anyway. So in order to avoid the sulfur dioxide, the coal must go through the desulfurization treatment.

Thirdly, the transformation from the coal to gas, although as the major energy of people`s production and life, but the use of the coal is not convenient enough,the coal has a relatively big size, it costs a lot of labor and material resources. We can transform the coal to gas by distillation, There is a lot of advantage of gas, it can burn sufficiently, and create more energy, it save spaces as well.

\section{Design of the processing mechanic equipment}

The major measure of the coal extraction and reprocessing includes: coal's selection,sulfur remove process and carbonization to produce gas. So, the mechanic equipment design should start from the three points mentioned above.

\section{Design of the mechanic equipment of coal selecting}

Coal selecting usually adopts mass method, in most cases, the heavy coal have less density, and the hierarchy inside is relatively higher,so the burning effect is better. So, one of the design principle of the coal selecting is the centrifugal force. Generate strong centrifugal force by high-speed electromotor, and then analysis according to the mechanical principle, the light coal will be sorted out, and the rest would be the heavy. The most common coal selecting equipment called coal selecting machine, its main function principal is the centrifugal theory, the core part is the circular vibrating screen, and select the coral by vibration and centrifugal theory.

\section{Design of the mechanic equipment of the coal desulfurization}

The coal desulfurization adopts chemical treatment methods, which is different from the coal selecting, because it uses the physical methods. So, the mechanic equipment design need to take more factors into consideration. First, the desulfurization equipment must be able to bear the high temperature and high pressure; because the main purpose of the chemical reaction is to neutralize the sulfur elements, or remove the pyrite in coal mine. So, the design of the mechanic equipment is as follows: 
First, It is needed to design the basic reaction vessel. The desulfurization have certain chemical condition, and the reaction vessel is necessary. The vessel must be able to bear the high pressure and high temperature to meet the requirement of the reaction.

Secondly, during the reaction, the equipment must be anti-corrosion. The theory basis of the desulfurization process is to oxidizing the sulfur element and make it become the acid, after the $\mathrm{PH}$ adjustment, acid solution will be neutralized, then the sulfur will be removed, thus to make sure the sulfur element in the coal will be decreased.

Thirdly, the desulfurization must be controlled by advanced professional system, there must be a dosing system, the substance added into is mainly the neutralizing agent. And the dosing system generally is controlled by the single chip machine and PLC controlling system, thus to make sure the time and frequency of the dosing. Besides, there must be a protection equipment, it is a very important link. The security of the desulfurization reflected in the neutralizing effect, only the effective neutralizing measure can make sure the security of desulfurization.

\section{The design of the coal's distillation mechanic equipment}

Coal distillation can increase the coal`s burning efficiency, thus to make sure the usage rate of the coal. The distillation of the coal is to let the coal went through the furnace with high temperature and high pressure until it decompose the coal, thus to provide the valuable gas. The gas can be widely applied in people`s production and life, and it also enjoys a very wide recognition among the users. So, when designing the distillation mechanic equipment, we mainly need to take two problems into consideration.

Firstly, the security issue of the distillation; because the main product of the distillation is the gas, the pressure problems of the gas is the major factor that will threaten the security. Because the reaction vessel is working under the high temperature and high pressure circumstance, so the tightness of the gas must be take into consideration in case there might be an explode.

Secondly,the efficiency issue of the distillation. The major purpose of the distillation is to decomposite the coal into gas, and it is a physical sublimation process. And the efficiency of the sublimation is a key point worthy of analysis. The major deciding factor of efficiency is the equipment. Because there will be coke in the distillation process, so the efficiency analysis is necessary. Under whatever condition,could they make sure the effectiveness of the sublimation, thus to improve the efficiency of the gas production, and slove the problem. So. The distillation of the coal need to pay attention the temperature and tightness in case there might be any danger, in the designing process, the security is the most important factor.

\section{Conclusion}

Due to its special property, the coal can not be used directly. So,the extraction and reprocessing are needed before it can be applied. The extract of the coal mainly adopted physical methods. As for the harmful gases produced by the coal, chemical desulfurization is needed to reduce the harmful gas emission. After the distillation, the coal will be decomposed because of the high temperature and produce the gas. The burning efficiency of the gas is very high, thus to make sure the burning efficiency of the coal. In order to slove this problem, this paper discusses the design of the coal`s extraction and reprocessing mechanic equipment, starts from its principal, examines carefully about the technology and quality, clarifies the designing philosophy of the processing mechanic, thus to make sure the high efficiency of the coal`s burning.

\section{References}

[1]Xiao Hua, Wei Zhiquan: Study of the characteristics of oxygen adsorption in measuring the coal spontaneous combustion tendency by oxygen inhalation method,Coal technology, 2013(1): 37-39

[2]Xu Jun, Ma Dongdong, Wang Suping: The SWOT analysis and strategy of the low-carbon economy development in the coal corporations, resource exploration and market, 2013(2): 166-169 
[3]Fang Ke,Wu Guilan, Ma Shuai: Study of the optimization of the drilling pre-pumping coal seam gas CFD simulation parameters, 2012(10):4-6

[4]Zhang Yong, Hu Jingming, Li Hang, Lin Chengyang, Gao Weiliang, Su Xin: Heat exchanger structure improvement of a pipe based on the CFD technology, Journal of Hunan Industry University.

[5]He Peng, Wang Zhen: The supporting effect data simulation of a coal mine roadway in Huannan coal mine, Journal of North China Institute of water conservancy and hydroelectric power, 2012(5): 91-94 